\title{
Anterior Cervical Epidural Abscess: The Use of Intraoperative Spinal Sonography
}

\author{
John A. Feldenzer, M.D., David C. Waters, M.D., James E. Knake, M.D., and \\ Julian T. Hoff, M.D. \\ Department of Surgery, Section of Neurosurgery, and Department of Radiology, Section of Neuroradiology, University of \\ Michigan Medical Center, Ann Arbor, Michigan
}

Feldenzer JA, Waters DC, Knake JE, Hoff JT. Anterior cervical epidural abscess: The use of intraoperative spinal sonography. Surg Neurol 1986;25:105-8.

\begin{abstract}
A case of acute cervical epidural abscess is presented. The use of intraoperative spinal sonography is discussed as a valuable adjunct in the evaluation and treatment of these uncommon lesions.
\end{abstract}

KEY WORDS: Spinal epidural abscess; Intraoperative spinal sonography; Spinal cord; Quadriplegia

Spinal epidural abscesses are uncommon and they represent acute neurosurgical emergencies. Neurological outcome is directly proportional to the speed and accuracy of the diagnosis. Proposed mechanisms of development include direct extension from adjacent osteomyelitic vertebrae or hematogenous spread from a distant focus of infection. Most spinal epidural abscesses are located posteriorly in the thoracic and lumbar spine. Epidural abscesses in the cervical area are rare.

The use of real-time ultrasonography in the operating room has become an increasingly important imaging technique. It is useful to assess the location and extent of tumors and inflammatory masses of the spinal canal and spinal cord, particularly when the lesion is not obvious or completely exposed. To our knowledge, the use of intraoperative spinal sonography to locate an anterior cervical epidural abscess has not been reported previously.

\section{Case Report}

The patient, a 73-year-old, right-handed white woman, was healthy except for mild hypertension. There was no

Address reprint requests to: John A. Feldenzer, M.D., Department of Surgery, Section of Neurosurgery, 1405 E. Ann Street, Ann Arbor, Michigan 48109. history of immunodeficiency, diabetes mellitus, recent infection, or trauma. One year prior to admission, she experienced severe pain in the neck and was admitted to another institution. No evidence of radicular pain or neurologic deficit was noted. Radiographs of the cervical spine revealed chronic degenerative changes and spontaneous fusion of the $\mathrm{C}-5$ and $\mathrm{C}-6$ vertebral bodies. A diagnosis of a posterior, paravertebral abscess of the soft tissue was made. Treatment consisted of incision and drainage of the abscess and a 1-month course of parenteral antibiotics. Stapbylococcus aureus was isolated from the operative cultures. The patient did well and her cervical pain resolved.

She developed an acute recurrence of cervical pain a year later, 10 days prior to admission. Three days later, she was admitted to an outside facility because of intractable cervical pain. At that time, she was afebrile with a normal neurological examination. The erythrocyte sedimentation rate was elevated $(98 \mathrm{~mm} / \mathrm{hr})$ and the peripheral leucocyte count was $14,800 / \mu \mathrm{L}$ with a normal differential. Radiographs and computerized axial scanning of the cervical spine were significant only for bony degenerative changes. Four days later, she developed an acute exacerbation of cervical pain with paresthesias and weakness in all four extremities. Over the next 24 hours her sensory loss and weakness progressed and she developed urinary retention and ileus. She developed respiratory distress on the day of her transfer to the University of Michigan.

Initial evaluation, on admission in November 1984, revealed an elderly, obese, white woman iń acute respiratory distress. Vital signs were stable except for a respiratory rate of $38 / \mathrm{min}$. Neurological examination revealed a complete $\mathrm{C}-6$ motor level and a complete sensory level at C8-T1 bilaterally with partial sensory sparing in the $\mathrm{C}-7$ dermatome on the left. Muscular tone was absent in the lower extremities with no demonstrable reflexes or plantar responses. Superficial reflexes were absent. Reflexes in the upper extremities were symmetrical: The biceps reflexes were normal, whereas 
the triceps and brachioradialis reflexes were absent. Rectal tone was absent and saddle anesthesia was present.

Radiographs of the cervical spine showed no vertebral subluxation or collapse. Loss of the normal C5-C6 disc space with fusion at this level was noted. In addition, significant prevertebral soft tissue swelling was present (Figure 1).

Studies of the blood revealed a peripheral leucocyte count of $12,700 / \mu \mathrm{L}$ (normal $=4,000-10,000)$ with a left-shifted differential. Alkaline phosphatase was 301 $\mathrm{IU} / \mathrm{L}$ (normal $=30-96 \mathrm{IU} / \mathrm{L}$ ) and the erythrocyte sedimentation rate was $100 \mathrm{~mm} / \mathrm{hr}$ (normal = less than 20 $\mathrm{mm} / \mathrm{hr}$ ). Cerebrospinal fluid (CSF), obtained from a C1C2 puncture, was clear and colorless with 10 white blood cells per milliliter ( $86 \%$ lymphocytes) and nine red blood cells per milliliter. CSF glucose was $103 \mathrm{mg} / \mathrm{dl}$ with a simultaneous serum glucose of $185 \mathrm{mg} / \mathrm{dl}$. CSF protein

Figure 1. Preoperative lateral radiograph of the cervical spine. Degenerative changes with sclerotic autofusion of the CS-C6 vertebral bodies and prevertebral swelling at C4-C6.

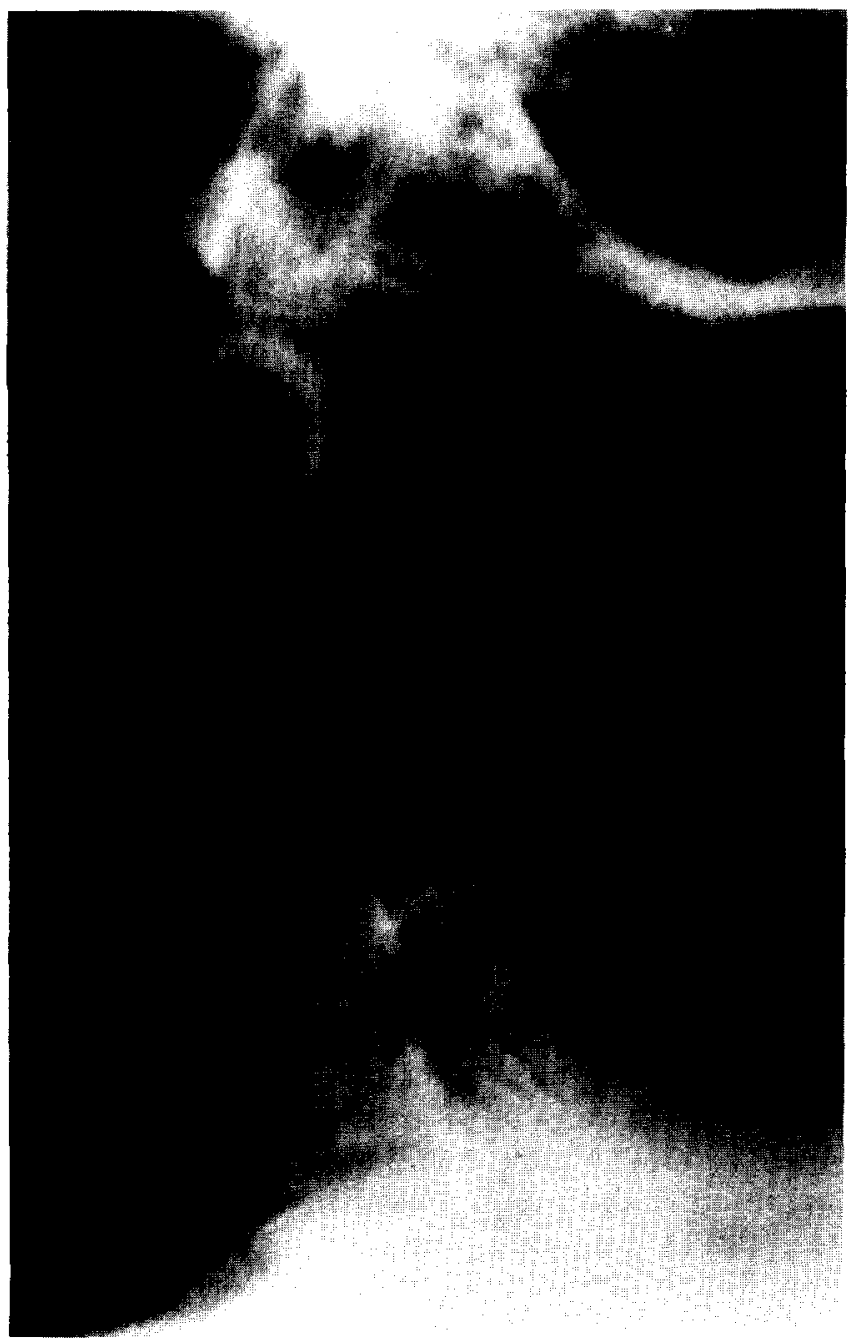

was $20 \mathrm{mg} / \mathrm{dl}$ (normal $=15-45 \mathrm{mg} / \mathrm{dl}$ ). CSF Gram stain, fungal smear, and cultures were subsequently negative.

Because of impending respiratory failure, the patient was intubated and stabilized in the emergency room. She then underwent a cervical metrizamide myelogram via a $\mathrm{C} 1-\mathrm{C} 2$ puncture in the supine position. A complete myelographic block with a large anterior extradural filling defect was noted at the C5-C6 level (Figure 2). The caudal extent of the block could not be ascertained myelographically or by computerized axial scanning with intrathecal contrast because of the tissue density similarity between the abscess and spinal cord.

Because of the progression of the patient's neurological deficit, an emergency $\mathrm{C} 3-\mathrm{C} 7$ posterior decompressive laminectomy was performed. Operative findings included a tense and nonpulsating dural sac, which was displaced posteriorly. No specific lesion could be identified. At that time, the real-time ultrasound scanner was used to locate an anterior lesion in longitudinal (Figure 3) and transverse planes (Figure 4). A 7.5-MHz transducer was utilized within a saline pool in the operative site. The anterior epidural space at C5-C6 was explored and a large amount of purulent material (10-15 $\mathrm{mL}$ ) was evacuated. Stapbylococcus aureus was isolated from intraoperative cultures. After the operation, the patient's neurological status remained unchanged. She was maintained on appropriate antibiotic coverage as dictated by culture and drug sensitivity results. She remained in the intensive care unit for $3 \frac{1}{2}$ months after the operation because of her dependence on a ventilator. She was subsequently transferred to the Physical Medicine and Rehabilitation Service and currently remains a C-6 quadriplegic.

\section{Discussion}

Cervical epidural abscesses, while rare, have been reported previously $[1,2,3,6,7,8,9,10,11,13,17,20,22]$. It has been suggested that the low incidence is due to the limitations of the cervical epidural space $[4,6,12]$. In the first complete description of the clinical and pathophysiologic aspects of spinal cord compression secondary to epidural abscess, Dandy stated that abscess formation does not occur in the cervical region, because of the anatomic limitation of the potential epidural space [4]. After studying the epidural space in cadavers, he stated that the size and the shape of this space is a function of the size of the spinal cord. He felt that the epidural space only becomes significant distal to the cervical enlargement.

Similarly, posterior epidural abscesses are more common than those in the anterior epidural space. This was also first noted by Dandy, who suggested that abscesses occurred more readily in the dorsal half of the canal 


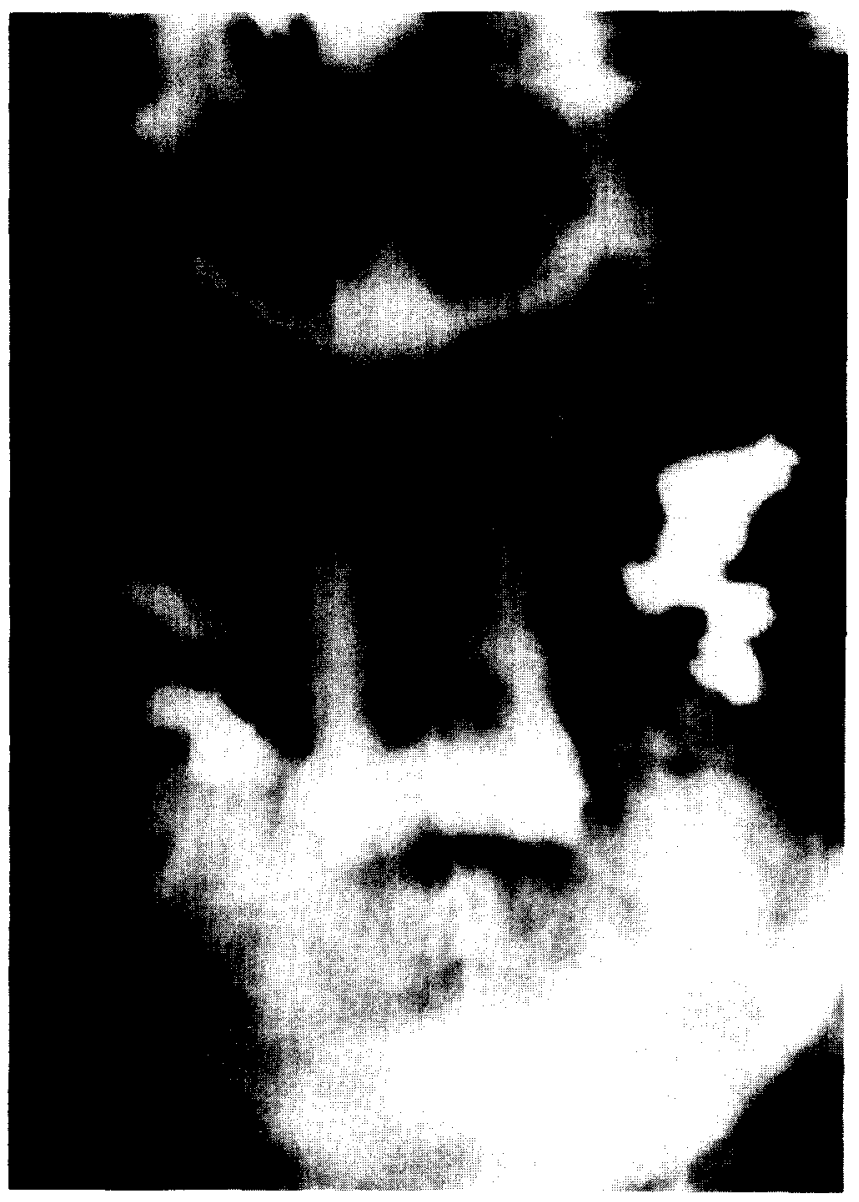

A

Figure 2. Supine preoperative cervical metrizamide myelogram via $C 1-C 2$ puncture. (A) Anteroposterior view demonstrating a complete block at the C5-C6 level. (B) Lateral view showing the anterior epidural mass (black arrow) at the C5-C6 level.

because of the close approximation of the dura mater to the vertebral bodies and ligaments anteriorly [4].

The etiology of spinal epidural abscess is attributed to direct extension from adjacent vertebral osteomyelitis or hematogenous spread from a distant focus of infection. Most anteriorly located abscesses are associated with adjacent vertebral osteomyelitis $[11,16]$. In cases where the etiology of cervical epidural abscesses is known, osteomyelitis is a more common cause than hematogenous spread $[6,10,11,12,16]$. This patient's abscess most likely developed from a chronic cervical osteomyelitis. Chronic changes consistent with osteomyelitis were noted on cervical radiographs 1 year earlier. The recent exacerbation of cervical pain with the development of progressive weakness, sensory loss, and sphincter dysfunction was due to an epidural abscess probably secondary to an indolent focus of vertebral osteomyelitis.

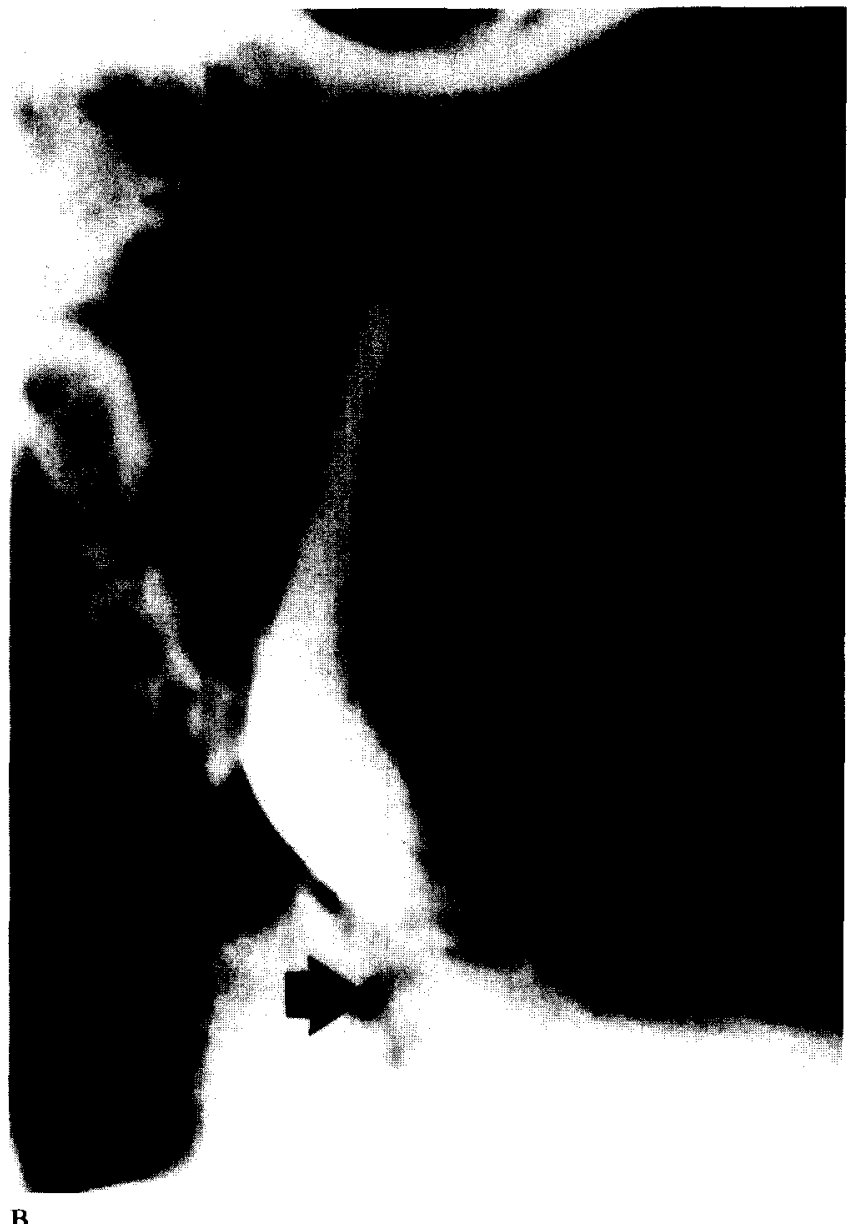

It was evident from the myelogram that a large anterior mass was present that extended over at least two vertebral segments. A cervical computerized axial scan immediately following myelography was not useful in defining the caudal extent of the mass. The tissue density similarity between the normal spinal cord and the extradural mass precluded differentiation by computed tomography. Usually this distinction can be made if tissue density differences, i.e., calcification, cyst, or fat, are sufficient to distinguish the spinal cord from the lesion. Therefore the caudal extent of the lesion could not be defined without performing a second myelogram in the lumbar region. This second myelogram was deferred because of the risk of passage of contrast material above the block into the cranial cavity when the contrast medium from below was moved upward. Because of the apparent size of the anterior mass and the inability to define its caudal extent, a multilevel cervical decompressive laminectomy was performed. Intraoperative spinal sonography clearly defined the cranial and caudal (as well as anteroposterior) extent of the abscess, thereby allowing complete decompression with minimal manipulation of the spinal cord. 


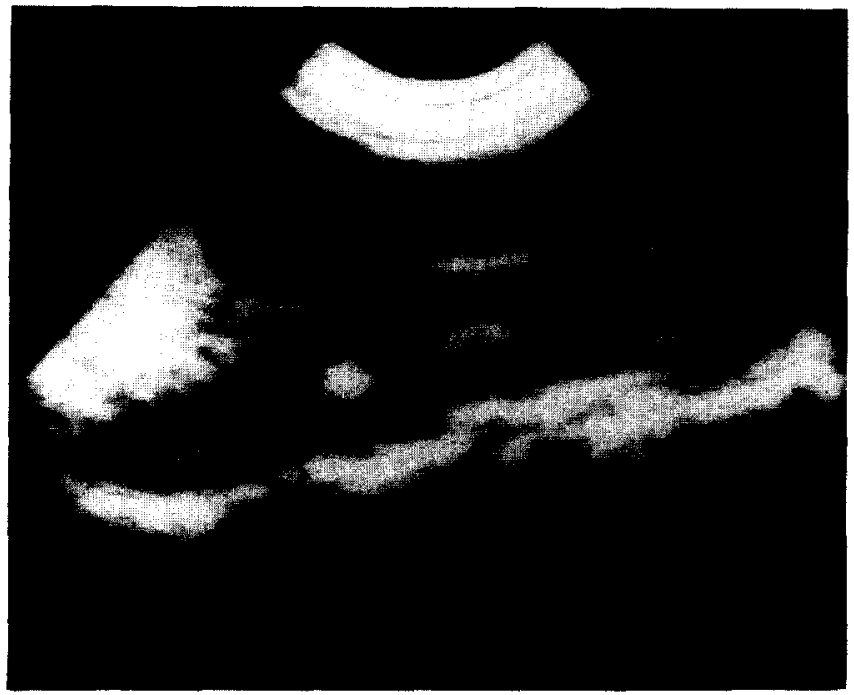

Figure 3. Intraoperative spinal sonography (sagittal view). Dorsal and ventral surfaces of the dura mater (small white arrows), anterior epidural mass (large white arrow), and posterior border of the vertebral bodies (open black arrow) are demonstrated. The ultrasonogram clearly demonstrates (1) the dorsal dural surface, (2) posterior displacement of a "sonographically normal" spinal cord with a linear central echo, (3) the anterior epidural mass, and (4) the posterior surface of the vertebral bodies.

The use of real-time sonography has proven to be effectve in defining the pathologic anatomy of extradural and intradural spinal masses $[5,14,15,18,19,21]$. The experience at the University of Michigan Medical Center with various spinal lesions has shown that a $7.5-\mathrm{MHz}$ scanning frequency through a saline pool is a technique that gives immediate and reliable information on the location, size, and presence of intradural or intramedullary lesions $[14,15]$. Sonography has been particularly useful in this and other cases of anteriorly located masses that are approached posteriorly $[14,15,18]$. It effectively allows for an accurate assessment of the location and size of an anterior lesion without retraction or manipulation of the spinal cord and with minimal bone resection. In addition, intraoperative sonography provides immediate information on the adequacy of the decompression.

\section{References}

1. Allbrook DB. Cervicodorsal osteomyelitis with extradural abscess. Lancet 1949;ii:1174-5.

2. Baker AS, Ojemann RG, Schwartz MN, Richardson EP Jr. Spinal epidural abscess. N Engl J Med 1975;293:463-8.

3. Browder J, Meyers R. Pyogenic infections of the spinal epidural space: a consideration of the anatomic and physiologic pathology. Surgery 1941;10:296-308.

4. Dandy WE. Abscesses and inflammatory tumors in the spinal epidural space (so-called pachymeningitis externa). Arch Surg 1926;13:477-94.

5. Dohrmann GJ, Rubin JM. Intraoperative ultrasound imaging of the spinal cord: syringomyelia, cysts and tumors-a preliminary report. Surg Neurol 1982;18:395-9.

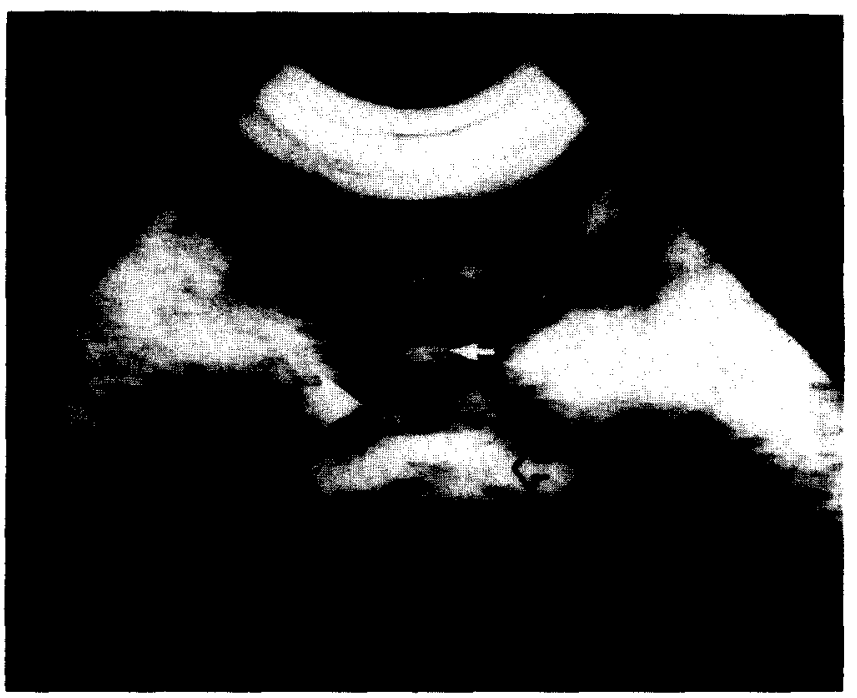

Figure 4. Intraoperative spinal sonograpby (transverse view). Dorsal and ventral surfaces of the dura mater (small white arrows), anterior epidural mass (large white arrow), and posterior border of the vertebral bodies (open black arrow) are demonstrated.

6. Durity F, Thompson GB. Localized cervical extradural abscess. J Neurosurg 1968;28:387-90.

7. Dus V. Spinal peripachymeningitis (epidural abscess). J Neurosurg 1960;17:972-83.

8. Gasul BM, Jaffe RH. Acute epidural spinal abscess-a clinical entity. Arch Pediatr 1935;52:361-90.

9. Hancock DO. A study of 49 patients with acute spinal extradural abscess. Paraplegia 1973;10:285-8.

10. Heusner AP. Nontuberculous spinal epidural infections. N Engl J Med 1948;239:845-54.

11. Hulme A, Dott NM. Spinal epidural abscess. Br Med J 1954;1:64-8.

12. Hutton PW. Acute osteomyelitis of cervical spine with epidural abscess. Br Med J 1956;1:153-4.

13. Kaufman DM, Kaplan JG, Litman N. Infectious agents in spinal epidural abscesses. Neurology 1980;30:844-50.

14. Knake JE, Chandler WF, McGillicuddy JE, Gabrielson TO, Latack JT, Gebarski SS, Yang PJ. Intraoperative sonography of intraspinal tumors: initial experience. AJNR 1983;4:1199-201.

15. Knake JE, Gabrielson TO, Chandler WF, Latack JT, Gebarski SS, Yang PJ. Real-time sonography during spinal surgery. Radiology 1984;151:461-5.

16. Mixter WJ, Smithwick RH. Acute intraspinal epidural abscess. N Engl J Med 1932;207:126-30.

17. Phillips GE, Jefferson AJ. Acute spinal epidural abscess: observations from fourteen cases. Postgrad Med 1979;55:712-5.

18. Quencer RM, Montalvo BM, Green BA, Eismont FJ. Intraoperative spinal sonography of soft tissue masses of the spinal cord and spinal canal. AJNR 1984;5:507-15.

19. Quencer RM, Montalvo BM. Normal intraoperative spinal sonography. AJNR 1984;5:501-5.

20. Ravicovitch MA, Spallone A. Spinal epidural abscesses: surgical and parasurgical management. Eur Neurol 1982;21:347-57.

21. Rubin JM, Dohrmann GJ. Work in progress: intraoperative ultrasonography of the spine. Radiology 1983;146:173-5.

22. Russell NA, Vaughan R, Morley TP. Spinal epidural infection. Can J Neurol Sci 1979;6:325-8. 